\title{
Evolutionary dynamics can be chaotic: A first example
}

Fabio Dercole (fabio.dercole@polimi.it)

Sergio Rinaldi (rinaldi@elet.polimi.it)

\section{Approved by}

Ulf Dieckmann

Program Leader, EEP

June 2011

Interim Reports on work of the International Institute for Applied Systems Analysis receive only limited review. Views or opinions expressed herein do not necessarily represent those of the Institute, its National Member Organizations, or other organizations supporting the work. 


\section{Contents}

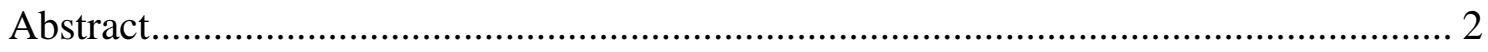

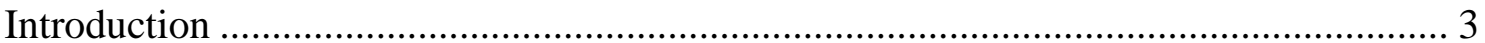

A tritrophic food chain model and its ad canonical equation ........................................ 5

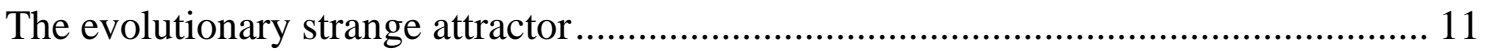

Feigenbaum cascade of period-doubling bifurcations .................................................. 12

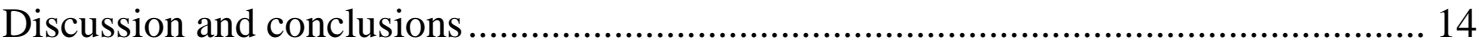

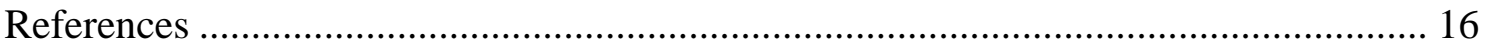

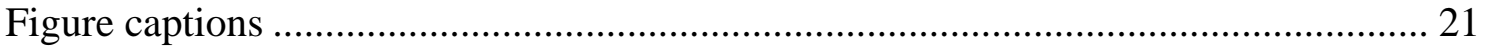

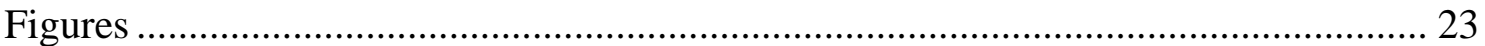




\title{
Evolutionary dynamics can be chaotic: A first example
}

\author{
Fabio Dercole ${ }^{1,3}$, Sergio Rinaldi ${ }^{1,2}$,
}

${ }^{1}$ DEI, Politecnico di Milano

Via Ponzio 34/5, 20133 Milano, Italy

${ }^{2}$ Evolution and Ecology Program, International Institute for Applied Systems Analysis 2361 Laxenburg, Austria

\footnotetext{
${ }^{3}$ To whom correspondence should be addressed

Ph: +3902 2399 3484; Fax: +39022399 3412

fabio.dercole@polimi.it
}

Running title: Evolutionary dynamics can be chaotic 


\begin{abstract}
We present in this paper the first example of chaotic evolutionary dynamics in biology. We consider a Lotka-Volterra tritrophic food chain composed of a resource, its consumer, and a predator species, each characterized by a single adaptive phenotypic trait, and we show that for suitable modeling and parameter choices the evolutionary trajectories approach a strange attractor in the three-dimensional trait space. The study is performed through the bifurcation analysis of the so-called canonical equation of Adaptive Dynamics, the most appropriate modeling approach to long-term evolutionary dynamics.
\end{abstract}

Key words: adaptive dynamics, deterministic chaos, evolution, population dynamics, strange attractor 


\section{Introduction}

Evolution by natural selection, as Darwin called it, is one of the oldest and most astounding and complex dynamical processes on Earth. Reduced to the essential, evolutionary change is the result of innovations and competition processes, the first altering heritable characteristics of individual agents and the second selecting the best performances. Genetic mutations and demographic competition play such roles in natural systems, but the scope of the Darwinian paradigm nowadays embraces many artificial systems in social sciences, economics, and engineering [Ziman, 2000].

The first mathematical investigations in evolutionary biology can be traced back to the field of population genetics [Fisher, 1930], classically focused on the change of the genotypic distribution of populations on a relatively short, demographic timescale. However, on a longer, evolutionary timescale, individuals interact according to the value of various adaptive phenotypic traits, the macroscopic manifestation of their microscopic genetic structure. The long-term evolution of such traits can be described to some extent by ignoring genetics. In fact, though sexual reproduction alters genotypic and therefore phenotypic distributions, mutations are the ultimate source of phenotypic variability, so that, in absence of mutation, the variances of trait distributions average out on a relatively short timescale. By looking at the long-term evolution of phenotypic traits, one can therefore imagine that male and female populations are monomorphic, i.e., phenotypically homogeneous, and produce either identical males and females or mutants. Given as granted that sex ratio remains constant, as is common practice in ecological modeling, one can measure population sizes in terms of males or females or, more abstractly, in terms of equivalent clonal organisms, whose genetics can be neglected.

Purely phenotypic, deterministic modeling approaches to long-term evolutionary dynamics have been proposed (see, e.g., [Brown \& Vincent, 1987; Hofbauer \& Sigmund, 1990; Christiansen, 1991; Vincent et al., 1993; Metz et al., 1996]), but one in particular, Adaptive Dynamics (AD) [Dieckmann \& Law, 1996; Metz et al., 1996; Geritz et al., 1997, 1998], explicitly links demographic dynamics, responsible for the selection pressure, to evolutionary changes. By considering mutations as small and rare events on the demographic timescale, AD describes evolution through an ordinary differential equation (ODE) on a separated, evolutionary timescale, in the space of the phenotypic traits characterizing the coevolving populations-the so-called AD canonical equation (see [Dercole \& Rinaldi, 2008] for a comprehensive treatment).

So far, the modeling effort has been limited to one or two evolving traits, for both application-specific 
arguments and mathematical tractability (see, e.g., [Abrams, 1992; Marrow et al., 1992; Dieckmann et al., 1995; Iwasa \& Pomiankowski, 1995; Marrow et al., 1996; Iwasa \& Pomiankowski, 1999; Abrams \& Matsuda, 1997; Gavrilets, 1997; Khibnik \& Kondrashov, 1997; Doebeli \& Ruxton, 1997; Kisdi et al., 2001; Dercole et al., 2002; Dercole, 2003; Dercole et al., 2003, 2006]). The most relevant conceptual result emerging from these studies is that reciprocal adaptation under constant environmental conditions can entrain two coevolving species in a perpetual cycle of coevolutionary change—the celebrated "Red Queen" dynamics, after [Van Valen, 1973]—in contrast with an optimization view of evolution. Although empirical evidence for Red Queen dynamics remains scant (with a few exceptions among paleontological time series [Soutar \& Isaacs, 1974; Baumgartner et al., 1992; Barnosky, 2001; Kelley et al., 2003] and well-controlled experiments on host-parasite and bacterial evolution [Dybdahl \& Storfer, 2003]), the conceptual possibility of non-stationary evolutionary regimes remains one of the "hot" topics in evolutionary theory. In particular, the following basic question spontaneously arises: Have evolutionary mechanisms involving a sufficiently large number of adaptive traits the power of generating deterministic chaos?

Of course, the answer couldn't come from previous modeling studies, due do the limited number of adaptive traits. The only attempt can be found in [Abrams \& Matsuda, 1997], where, assuming rapid evolution (behavioral adaptation or very high mutational rates), demographic and evolutionary dynamics were considered on the same timescale and turned out to be chaotic in a three-dimensional space composed of a single adaptive trait, characterizing a resource harvested by a non-adaptive consumer, and the abundances of resource and consumer populations. However, by allowing adaptive traits and population abundances to evolve on the same timescale, one cannot say if the complexity of the dynamics are the genuine consequence of innovation-competition processes or if they are induced by particular demographic interactions.

In principle, the answer to our basic question could be given if rich sets of field or laboratory data would be available. Unfortunately, time series of adaptive traits are typically too short (with the exception of the few paleontological time series and laboratory experiments mentioned above) to justify the use of the statistical techniques proposed in the last decades for revealing the existence of deterministic chaos [Ott et al., 1994; Abarbanel, 1996]. On the other hand, paleontological time series often refer to the evolution of systems driven by highly chaotic climatic variations. Therefore, the statistical tests applied to these series simply say that the biological response to climate variability is chaotic, but do not reveal, however, if evolutionary mechanisms have the power of generating their own chaos. By contrast, evolutionary models with constant parameters virtually mimic the ideal conditions of an absolutely not varying physical environment, and are 
therefore perfectly suited for answering our question. Moreover, given as granted that more details (for example, on sex or age, stages, and space structures) will increase the chances of generating chaos in a model, we can reasonably pretend to positively answer the question if we can show that a simple threedimensional evolutionary model can be chaotic. This is, actually, what has been done in various fields of science, starting with meteorology [Lorenz, 1963], where the first strange attractor has been found, and proceeding with mechanics [Hayashi et al., 1970], chemistry [Rössler, 1976], electronics [Madan, 1993], epidemiology [Schwartz \& Smith, 1983], and ecology [Hastings \& Powell, 1991]. Of course, we should a priori expect a positive answer if we believe that evolution is at least as complex as the just mentioned fields.

This paper is devoted to the presentation of the first chaotic evolutionary attractor, obtained through the $\mathrm{AD}$ approach. In the next section, a very simple tritrophic food chain is considered and the corresponding three-dimensional AD canonical equation is derived. Then, in the following section it is shown that for a suitable parameter setting the evolutionary dynamics are chaotic, and the main characteristics of the evolutionary strange attractor are analyzed. In Sect. 4 it is also shown how the evolutionary regimes change from stationary to cyclic and then from cyclic to chaotic when the mutational rate of the population at the lowest trophic level is increased. This points out the most frequent "route to chaos," namely the famous Feigenbaum cascade of period-doubling bifurcations [Feigenbaum, 1980]. A few comments on the value and limitations of our findings close the paper.

As for the style of the paper, we mix a somewhat naïve description of the underlying biological processes with a tutorial presentation of some mathematical concepts. This choice, already reflected in this Introduction, makes the paper accessible and useful to a broader class of readers, from pure and applied mathematicians to theoretically inclined biologists, as well as to scientists in various fields of science and engineering.

\section{A Tritrophic Food Chain Model and Its AD Canonical Equation}

The demographic model we use for pointing out evolutionary chaos is the classical Lotka-Volterra tritrophic food chain composed of resource, consumer, and predator populations, each characterized by a single adaptive trait. There are three reasons for this choice. First, the model is very simple and therefore appropriate for investigating the possibility of chaotic evolutionary regimes. Second, on the short-term demographic timescale, i.e., for given values of the traits, the three populations can only coexist at a unique and stable 
demographic equilibrium. This simplifies the analysis, since the AD canonical equation is formally derived for the case of stationary demographic coexistence (see [Dercole et al., 2006] for discussions and extensions), but also strengthens our answer, by showing that evolution can be chaotic without requiring wild demographic dynamics of the populations. Third, the model is the natural extension of the ditrophic food chain where evolutionary cycles were first observed [Dieckmann et al., 1995] (see also [Dercole et al., 2003] for further analysis). This suggests that a two-species model with cyclic evolutionary regimes could easily become chaotic by adding a third coevolving species.

The Lotka-Volterra tritrophic food chain model has the form

$$
\begin{aligned}
& \dot{n}_{1}=r\left(x_{1}\right) n_{1}-c\left(x_{1}\right) n_{1}^{2}-a_{2}\left(x_{1}, x_{2}\right) n_{1} n_{2}, \\
& \dot{n}_{2}=e_{2} a_{2}\left(x_{1}, x_{2}\right) n_{1} n_{2}-d_{2}\left(x_{2}\right) n_{2}-a_{3}\left(x_{2}, x_{3}\right) n_{2} n_{3}, \\
& \dot{n}_{3}=e_{3} a_{3}\left(x_{2}, x_{3}\right) n_{2} n_{3}-d_{3}\left(x_{3}\right) n_{3},
\end{aligned}
$$

where $n_{1}, n_{2}$, and $n_{3}$ are the resource, consumer, and predator population abundances, $r$ and $c$ are resource net growth rate and intraspecific competition, and $a_{i}, e_{i}$, and $d_{i}$ are attack rate, efficiency, and net death rate (not due to consumption) of consumer $(i=2)$ and predator $(i=3)$. Three adaptive traits, one per population, $x_{1}, x_{2}$, and $x_{3}$, characterize the food chain, in the sense that inter- and intra-specific demographic parameters depend upon the traits of the involved populations. In view of the importance of individual body size in determining demographic interactions between resources and consumers [Cohen et al., 1993], we imagine $x_{i}, i=1,2,3$, as real variables obtained from the body sizes of adult individuals through suitable nonlinear scalings.

Model (1) has a unique nontrivial equilibrium

$$
\begin{aligned}
& \bar{n}_{1}=\frac{1}{c}\left(r-\frac{a_{2} d_{3}}{e_{3} a_{3}}\right), \\
& \bar{n}_{2}=\frac{d_{3}}{e_{3} a_{3}}, \\
& \bar{n}_{3}=\frac{e_{2} a_{2}}{a_{3} c}\left(r-\frac{a_{2} d_{3}}{e_{3} a_{3}}\right)-\frac{d_{2}}{a_{3}},
\end{aligned}
$$


which is positive if and only if $\bar{n}_{3}>0$, i.e.,

$$
\frac{r}{c}-\frac{a_{2} d_{3}}{c e_{3} a_{3}}-\frac{d_{2}}{e_{2} a_{2}}>0 .
$$

Moreover, the equilibrium (2) is always globally stable (in the positive orthant $n_{i} \geq 0, i=1,2,3$ ), which means that under condition (3) the model has only one asymptotic mode of behavior, namely stationary coexistence. Condition (3) with the equality sign marks the extinction of the predator population and technically corresponds to a transcritical bifurcation of model (1), at which the equilibrium (2) collides and exchanges stability with the trivial equilibrium lying on the face $n_{3}=0$ of the demographic state space.

Model (1) is called the resident model, because, under condition (3), it allows the coexistence of the three considered populations, which are therefore called resident. More precisely, to specify the resident model, we must say how the demographic parameters depend upon the traits. The number of possibilities is practically unlimited because even for well-identified species there are many meaningful options. To be consistent with the analysis of the ditrophic food chain carried out in [Dieckmann et al., 1995; Dercole et al., 2003], we assume that the parameters $r, e_{i}$, and $d_{i}, i=1,2$, are trait-independent, while resource intraspecific competition $c$ is given by

$$
c\left(x_{1}\right)=c_{1}+c_{2}\left(x_{1}-c_{0}\right)^{2},
$$

where parameter $c_{0}$ is the optimum resource trait at which intraspecific competition is minimum, and the attack rates $a_{2}$ and $a_{3}$ are

$$
\begin{aligned}
& a_{2}\left(x_{1}, x_{2}\right)=\exp \left(-\left(\frac{x_{1}-a_{24}}{a_{21}}\right)^{2}+2 a_{23} \frac{\left(x_{1}-a_{24}\right)\left(x_{2}-a_{25}\right)}{a_{21} a_{22}}-\left(\frac{x_{2}-a_{25}}{a_{22}}\right)^{2}\right) \\
& a_{3}\left(x_{2}, x_{3}\right)=\exp \left(-\left(\frac{x_{2}-a_{34}}{a_{31}}\right)^{2}+2 a_{33} \frac{\left(x_{2}-a_{34}\right)\left(x_{3}-a_{35}\right)}{a_{31} a_{32}}-\left(\frac{x_{3}-a_{35}}{a_{32}}\right)^{2}\right)
\end{aligned}
$$

where $a_{23}<1$ and $a_{33}<1$. If resource and consumer [consumer and predator] traits are tuned, i.e., if $x_{1}=a_{24}, x_{2}=a_{25}\left[x_{2}=a_{34}, x_{3}=a_{35}\right.$, the consumer [predator] attack rate is maximum. When resource and consumer [consumer and predator] traits are far from being tuned, the consumer [predator] attack rate vanishes.

We are now ready to derive the evolutionary dynamics, namely the dynamics of the traits $x_{1}, x_{2}$, and 
$x_{3}$ on the evolutionary timescale. Obviously, evolutionary trajectories are defined in the open region of trait space where condition (3) is satisfied. This region is called the evolution set, denoted by $\mathcal{X}$, and results in the bounded volume shown in Fig. 1A. Due to its ovoid shape, we like to call it "the egg of life". Evolutionary trajectories are therefore defined inside the egg, as exemplified in panel B, where evolution dynamics halt at a stable evolutionary equilibrium. Along an evolutionary trajectory the food chain coexists at the demographic equilibrium (2), so that resource, consumer, and predator abundances slowly vary, on the evolutionary timescale, by following the equilibrium values (2) at the current evolutionary state.

Evolutionary change of trait $x_{i}$ result from a sequence of successful mutations, after which the mutant population, characterized by a trait value $x_{i}^{\prime}$ slightly different from the resident value $x_{i}$, substitutes the corresponding resident population. This demographic transient takes an infinitesimal time on the evolutionary timescale and marks an infinitesimal step in the evolution of trait $x_{i}$, i.e., $x_{i} \rightarrow x_{i}^{\prime}$. Since mutations are assumed as rare events on the demographic timescale, we can consider one mutation at a time. Then $\mathrm{AD}$ smooths the innovation-competition process, which is intrinsically stochastic, and describes the average evolutionary trajectory by means of the canonical equation.

More in particular, if we imagine that a mutant population is also present, we must enlarge model (1) by adding a fourth ODE for the mutant population and by specifying how the demographic parameters depend upon the traits $x_{1}, x_{2}, x_{3}, x_{i}^{\prime}$. In the case of a mutation in the resource population, the resident-mutant model is

$$
\begin{aligned}
& \dot{n}_{1}=n_{1}\left(r-c\left(x_{1}\right) n_{1}-\gamma\left(x_{1}, x_{1}^{\prime}\right) n_{1}^{\prime}-a_{2}\left(x_{1}, x_{2}\right) n_{2}\right), \\
& \dot{n}_{2}=n_{2}\left(e_{2} a_{2}\left(x_{1}, x_{2}\right) n_{1}+e_{2} a_{2}\left(x_{1}^{\prime}, x_{2}\right) n_{1}^{\prime}-d_{2}-a_{3}\left(x_{2}, x_{3}\right) n_{3}\right), \\
& \dot{n}_{3}=n_{3}\left(e_{3} a_{3}\left(x_{2}, x_{3}\right) n_{2}-d_{3}\right), \\
& \dot{n}_{1}^{\prime}=n_{1}^{\prime}\left(r-\gamma\left(x_{1}^{\prime}, x_{1}\right) n_{1}-c\left(x_{1}^{\prime}\right) n_{1}^{\prime}-a_{2}\left(x_{1}^{\prime}, x_{2}\right) n_{2}\right),
\end{aligned}
$$

where $\gamma\left(x_{1}, x_{1}^{\prime}\right)$ is the competition coefficient characterizing reduced birth rate and/or increased death rate in the resource resident population due to the competition with the resource mutant population (necessarily $\left.\gamma\left(x_{1}, x_{1}\right)=c\left(x_{1}\right)\right)$. In agreement with classical competition theory (see, e.g., [Gatto, 1990]), competition is said to be symmetric if the competition function $\alpha\left(x_{1}, x_{1}^{\prime}\right)=\gamma\left(x_{1}, x_{1}^{\prime}\right) / c\left(x_{1}\right)$ is unaffected by the exchange of its arguments, i.e., $\alpha\left(x_{1}, x_{1}^{\prime}\right)=\alpha\left(x_{1}^{\prime}, x_{1}\right)$, asymmetric otherwise. As in [Dieckmann et al., 1995; Dercole et al., 2003], we assume a constant (therefore symmetric) competition function, so that $\gamma\left(x_{1}, x_{1}^{\prime}\right)=c\left(x_{1}\right)$. 
Similarly, the two other resident-mutant models, describing the demographic interactions in the cases of mutations in the consumer and in the predator populations, are given by

$$
\begin{aligned}
& \dot{n}_{1}=n_{1}\left(r-c\left(x_{1}\right) n_{1}-a_{2}\left(x_{1}, x_{2}\right) n_{2}-a_{2}\left(x_{1}, x_{2}^{\prime}\right) n_{2}^{\prime}\right), \\
& \dot{n}_{2}=n_{2}\left(e_{2} a_{2}\left(x_{1}, x_{2}\right) n_{1}-d_{2}-a_{3}\left(x_{2}, x_{3}\right) n_{3}\right), \\
& \dot{n}_{3}=n_{3}\left(e_{3} a_{3}\left(x_{2}, x_{3}\right) n_{2}+e_{3} a_{3}\left(x_{2}^{\prime}, x_{3}\right) n_{2}^{\prime}-d_{3}\right), \\
& \dot{n}_{2}^{\prime}=n_{2}^{\prime}\left(e_{2} a_{2}\left(x_{1}, x_{2}^{\prime}\right) n_{1}-d_{2}-a_{3}\left(x_{2}^{\prime}, x_{3}\right) n_{3}\right),
\end{aligned}
$$

and

$$
\begin{aligned}
& \dot{n}_{1}=n_{1}\left(r-c\left(x_{1}\right) n_{1}-a_{2}\left(x_{1}, x_{2}\right) n_{2}\right), \\
& \dot{n}_{2}=n_{2}\left(e_{2} a_{2}\left(x_{1}, x_{2}\right) n_{1}-d_{2}-a_{3}\left(x_{2}, x_{3}\right) n_{3}-a_{3}\left(x_{2}, x_{3}^{\prime}\right) n_{3}^{\prime}\right), \\
& \dot{n}_{3}=n_{3}\left(e_{3} a_{3}\left(x_{2}, x_{3}\right) n_{2}-d_{3}\right), \\
& \dot{n}_{3}^{\prime}=n_{3}^{\prime}\left(e_{3} a_{3}\left(x_{2}, x_{3}^{\prime}\right) n_{2}-d_{3}\right) .
\end{aligned}
$$

At this point, the $\mathrm{AD}$ canonical equation can be derived. A crucial quantity in modeling evolutionary dynamics is the so-called invasion fitness of a mutation [Metz et al., 1992], namely the initial exponential rate of growth of the mutant population. This is technically given by the dominant eigenvalue associated to the equilibrium $\left(\bar{n}_{1}, \bar{n}_{2}, \bar{n}_{3}, 0\right)$ of the resident-mutant model (after linearization), the dominant eigenvector being the only one with nontrivial mutant component. Thus, positive fitness means mutant invasion, while negative fitness characterizes unsuccessful mutations. Straightforward computations yield the following invasion fitnesses for the resource, consumer, and predator mutant populations:

$$
\begin{aligned}
& \lambda_{1}\left(x_{1}, x_{2}, x_{3}, x_{1}^{\prime}\right)=r-c\left(x_{1}^{\prime}\right) \bar{n}_{1}\left(x_{1}, x_{2}, x_{3}\right)-a_{2}\left(x_{1}^{\prime}, x_{2}\right) \bar{n}_{2}\left(x_{1}, x_{2}, x_{3}\right), \\
& \lambda_{2}\left(x_{1}, x_{2}, x_{3}, x_{2}^{\prime}\right)=e_{2} a_{2}\left(x_{1}, x_{2}^{\prime}\right) \bar{n}_{1}\left(x_{1}, x_{2}, x_{3}\right)-d_{2}-a_{3}\left(x_{2}^{\prime}, x_{3}\right) \bar{n}_{3}\left(x_{1}, x_{2}, x_{3}\right), \\
& \lambda_{3}\left(x_{1}, x_{2}, x_{3}, x_{3}^{\prime}\right)=e_{3} a_{3}\left(x_{2}, x_{3}^{\prime}\right) \bar{n}_{2}\left(x_{1}, x_{2}, x_{3}\right)-d_{3},
\end{aligned}
$$

where the equilibrium abundances $\bar{n}_{i}\left(x_{1}, x_{2}, x_{3}\right), i=1,2,3$, are given by (2). Then, the $\mathrm{AD}$ canonical 
equation is obtained as

$$
\dot{x}_{i}=\left.k_{i} \bar{n}_{i}\left(x_{1}, x_{2}, x_{3}\right) \frac{\partial}{\partial x_{i}^{\prime}} \lambda_{i}\left(x_{1}, x_{2}, x_{3}, x_{i}^{\prime}\right)\right|_{x_{i}^{\prime}=x_{i}}, \quad i=1,2,3
$$

where $k_{i}=1 / 2 \mu_{i} \sigma_{i}^{2}, i=1,2,3$, are constant mutational rates, proportional to the frequency $\left(\mu_{i}\right)$ and variance $\left(\sigma_{i}^{2}\right)$ of mutations in the three populations, and

$$
\left.\frac{\partial}{\partial x_{i}^{\prime}} \lambda_{i}\left(x_{1}, x_{2}, x_{3}, x_{i}^{\prime}\right)\right|_{x_{i}^{\prime}=x_{i}}
$$

called the selection derivative, gives the direction and strength of the selection pressure on trait $x_{i}$ (note that $\dot{x}_{i}$ denotes time derivative on the evolutionary timescale).

Without going into the detail of the derivation, we can say that the rate of evolutionary change $\dot{x}_{i}$ is influenced by three primary factors: how often a mutation occurs in population $i, \mu_{i} \bar{n}_{i}$ is in fact proportional to the number of mutations occurring in population $i$ per unit of evolutionary time; how large is the trait change entailed by the mutation, zero mean and variance $\left(\epsilon \sigma_{i}\right)^{2}, \epsilon$ being a scaling factor separating the demographic and evolutionary timescales in the limit $\epsilon \rightarrow 0$; and how likely it is that the initially scarce mutant population invades and replaces the corresponding resident population. The probability of invasion consists of two factors. First, if the selection derivative (5) does not vanish, only mutations with trait value either larger or smaller than the resident value can invade; in other words, half of the mutations are at selective disadvantage and this leads to the factor $1 / 2$ in the canonical equation. Second, mutations at selective advantage may be accidentally lost in the initial phase of invasion when they are present only in a few items. The probability of success in the initial phase of invasion is shown to be proportional to the selective advantage of the innovation, as measured by the selection derivative. Finally, successful invasion generically implies substitution (see [Dercole \& Rinaldi, 2008, Chap. 3] for a detailed derivation). The explicit expression of the canonical equation (4) is not reported because long and tedious. However, it can be easily generated and handled by means of symbolic computation.

The canonical equation (4) can be studied through bifurcation analysis. However, this would require notions of bifurcation theory that are not available to many biologists. For this reason equation (4) will first be used to carry out selected simulations, namely to determine the evolution of the three traits for given parameter settings and for given ancestral conditions $x_{i}(0), i=1,2,3$. Of course, the ancestral 
conditions must guarantee the demographic coexistence of the three populations, i.e., $\left(x_{1}(0), x_{2}(0), x_{3}(0)\right)$ must be in the evolution set $\mathcal{X}$ (see Fig. 1A). The simulation of equation (4), starting from a viable initial point, produces an evolutionary trajectory, which can either remain in $\mathcal{X}$ forever, thus converging toward an evolutionary attractor (an equilibrium in the case of Fig. 1B), or reach the boundary of $\mathcal{X}$ in finite time, thus inducing the evolutionary extinction [Ferrière, 2000] of the predator population. This is actually a case of "evolutionary murder", since the boundary of $\mathcal{X}$ is a transcritical bifurcation of the resident model (1), at which the predator population abundance $\bar{n}_{3}$ annihilates, so that also the predator evolutionary rate of change $\dot{x}_{3}$ vanishes while approaching extinction (see (4)). The boundary of $\mathcal{X}$ is therefore reached thanks to the evolution of the resource and consumer populations, which play the role of murderers.

\section{The Evolutionary Strange Attractor}

We now present an evolutionary strange attractor produced by the $\mathrm{AD}$ canonical equation (4). As far as we know, this is the first example of chaotic attractor describing long-term evolutionary dynamics. For the moment we do not reveal how we were able to find the corresponding parameter setting, but a discussion on this point is reported in the next section.

The strange attractor is shown in Fig. 2. The left panel (Fig. 2A) points out the attractor in the threedimensional trait space, while the right panel (Fig. 2B) shows three segments of the corresponding aperiodic time series $x_{i}(t), i=1,2,3$. Some features of the attractor can be identified through visual inspection. Indeed, Fig. 2B shows that the traits of the first two species vary almost periodically, while $x_{3}$ varies more irregularly. The intervals between successive peaks of the predator trait $x_{3}$ are almost constant (high coherence), while the peaks of $x_{3}$ alternate irregularly, like in the classical two-band Rössler attractor [Rössler, 1976]. Figure 2A shows that the attractor lies roughly on a Möbius strip and has therefore a fractal dimension very close to 2 . Since strange attractors with fractal dimension close to 2 must have "peak-to-peak dynamics" [Candaten \& Rinaldi, 2000], one should a priori expect that the so-called peak-to-peak plot, namely the set of all pairs of successive peaks of any trait, identifies a smooth curve. This is indeed the case, as shown in Fig. 3, where each point represents a pair of successive peaks of the predator trait $x_{3}$ extracted from a long time series produced by model (4). The curve drawn in Fig. 3, called the skeleton of the peak-to-peak plot, can be used to predict the value of the next peak of the predator trait from the value of the last peak (a similar prediction can be made on the evolutionary time of occurrence of the next peak). This is a rather 
intriguing property, since it shows that at least some predictions are possible even if the system is chaotic.

Three Lyapunov exponents, $L_{1}>L_{2}>L_{3}$, are associated with the strange attractor of Fig. 2. Lyapunov exponents measure the mean exponential rates of initial divergence (if positive) or convergence (if negative) of nearby initial conditions along three independent directions suitably selected at each point of the attractor (see, e.g., [Ramasubramanian \& Sriram, 2000] for computational issues). Chaotic attractors are characterized by both positive and negative Lyapunov exponents, determining divergence (stretching) of nearby initial conditions and convergence (folding) to the attractor. Moreover, one exponent is always zero (the trivial exponent), since exponential divergence and convergence do not affect initial conditions on the same trajectory. Thus, it must be $L_{1}>0, L_{2}=0, L_{3}<0$, where the largest Lyapunov exponent $L_{1}$ measures the mean exponential rate of initial divergence of two generic nearby points on the attractor, i.e., the mean sensitivity to initial conditions. The estimates obtained with the so-called standard algorithm [Ramasubramanian \& Sriram, 2000] are

$$
L_{1}=8.1321 \times 10^{-3}, \quad L_{2}=-2.3923 \times 10^{-6}, \quad L_{3}=-4.6270 \times 10^{-1},
$$

and the corresponding attractor fractal dimension, obtained with the Kaplan-Yorke formula $\left(2-L_{1} / L_{3}\right)$ [Alligood et al., 1996], is 2.0176, which confirms the correctness of our visual interpretation of Fig. 2A.

\section{Feigenbaum Cascade of Period-doubling Bifurcations}

We now explain how we arrived at the evolutionary strange attractor described in the previous section. Hunting for strange attractors is a very peculiar game and the worst possible way to play this game is to do it randomly. One should use instead a mix of intuition and theory.

In the present case intuition was based on the results obtained in [Dieckmann et al., 1995; Dercole et al., 2003], where it was shown that one way of obtaining the most complex evolutionary dynamics (cyclic dynamics in that case) of a ditrophic food chain composed of resource and consumer was to increase the mutational rate of the resource. We could then retain that message, and hope, on a purely intuitive ground, that the resource mutational rate could be an effective control parameter for transforming simple (i.e., stationary) into complex (i.e., chaotic) evolutionary dynamics in tritrophic food chains. On the other hand, bifurcation theory is quite precise on this matter. It says that there are two common routes to chaos, namely 
two special sequences of bifurcations that characterize successive structural changes of the attractor, until the strange attractor appears. One route is marked by a particular bifurcation, called Shil'nikov homoclinic bifurcation, and the other is the celebrated Feigenbaum cascade of period-doubling bifurcations.

For biologists who are not aware of period-doubling bifurcations and Feigenbaum cascade, we can say that, physically speaking, a period-doubling (also called flip) bifurcation occurs when a small variation of a parameter $p$, from $p_{1}-\epsilon$ to $p_{1}+\epsilon$, transforms an attracting cycle of period $T$ into an attractive cycle of period $2 T$, as shown in Fig. 4. A second period-doubling bifurcation at $p=p_{2}$ would transform the cycle of Fig. 4B into a cycle with four loops, and so on. Of course, the period of the cycle at $p=p_{2}-\epsilon$ might be radically different than $2 T$, though in technical jargon one often says that the second period-doubling yields a "period-4" cycle. Thus, $n$ successive period-doubling bifurcations $\left\{p_{1}, p_{2}, \ldots, p_{n}\right\}$ transform the period1 cycle into a period- $2^{n}$ cycle. The Feigenbaum cascade is an infinite sequence $\left\{p_{i}\right\}$ of period-doubling bifurcations in which the bifurcation values $p_{i}$ accumulate at a critical value $p_{\infty}$ after which the attractor is a genuine strange attractor. Very often, the strange attractor is coherent and its shape is similar to the period-1 cycle that has originated the whole cascade [Alligood et al., 1996].

With these elements in mind, we started from a parameter setting giving rise to an evolutionary equilibrium, and tried to obtain an evolutionary cycle by varying some of the parameters. For doing this, we took into account the analysis performed in [Dercole et al., 2003], which suggests parameter settings giving rise to cyclic dynamics in ditrophic food chains. Thus, once we had an evolutionary cycle we increased our candidate control parameter (the resource mutational rate $k_{1}$ ) and after a few trials we were able to detect a first period-doubling bifurcation, i.e., a clear warning of a possible route to chaos. A further increase of the control parameter has confirmed the existence of a Feigenbaum cascade and has finally produced the strange attractor of Fig. 2. This route to chaos is visualized in Fig. 5, where the six panels A-F show the attractors corresponding to increasing values of the control parameter $p$, namely $k_{1}$ : A, equilibrium; $\mathrm{B}$, small cycle originated through a Hopf bifurcation at $p=p_{H}$; C, large cycle; D, cycle after the first period-doubling, $p_{1}<p<p_{2}$; E, cycle after the second period-doubling, $p_{2}<p<p_{3}$; F, strange attractor, $p>p_{\infty}$ (note that the evolution set $\mathcal{X}$ is unaffected by $k_{i}, i=1,2,3$, see (3)). Figure 6 reports the standard representation of the Feigenbaum cascade, where the peaks of $x_{3}$ within the attractor are plotted for each value of the control parameter.

The shape of our strange attractor (see Fig. 2) clearly reveals its Feigenbaum origin. Indeed, in the chaotic regime, the first two traits oscillate almost periodically at the frequency $1 / T$, while the third trait 
oscillates more irregularly but still at the same average frequency (coherence). A physical image can be associated with the chaotic evolutionary dynamics we have detected: one simply needs to stand on a beach and observe the waves, which, indeed, arrive quite regularly on the beach but with irregular (and often alternating) heights like the waves of the predator trait.

To complement the one-parameter analysis presented in Fig. 6, we report in Fig. 7 a two-parameter bifurcation diagram, where the range of interest for the ratios $k_{1} / k_{3}$ and $k_{2} / k_{3}$ is fixed to $[0.2,2]$ (note that $k_{3}$ can be set to 1 by scaling evolutionary time). Black lines represent bifurcation curves, obtained through numerical continuation (see, e.g., [Allgower \& Georg, 1990]), the most effective and simulation-free method for analyzing equilibria and cycles under parameter perturbation (the standard continuation package AUTO was used [Doedel et al., 2007]). The curve separating the white from the color-coded region in the main (left) panel is the Hopf bifurcation, while the other curves identify, from left to right, the first four perioddoubling bifurcations of the Feigenbaum cascade (the third and fourth bifurcations are distinguishable only at the scale of the enlargement in the right panel). Thus, the evolutionary regime is stationary for parameter combinations corresponding to a point in the white region and non-stationary (Red Queen dynamics) for combinations corresponding to a color in the color-bar, the color indicating the maximum (nontrivial) Lyapunov exponent. Green and red tones therefore indicate stable cycles and strange attractors, respectively (for purpose of illustration, the color-bar linearly maps colors to the intervals of negative and positive exponents, separately). For parameter combinations in the gray region, the evolutionary extinction of the predator population is the inevitable outcome, since the evolutionary strange attractor grazes the boundary of the evolution set $\mathcal{X}$ and gets destroyed at the separation between the reddish and the gray region.

\section{Discussion and Conclusions}

We have shown in this paper that the study of a Lotka-Volterra three-species food chain reveals the possibility of chaotic evolutionary dynamics. This confirms that a possible consequence of innovation-competition (mutation-selection) processes is that species coevolve in an apparently random fashion.

The very special properties of our evolutionary strange attractor (coherence and peak-to-peak dynamics) are certainly due to the extreme simplicity of the model and would probably be lost with the addition of some extra realism. In other words, we believe that the study of evolving systems with more complex structures would reveal more complex chaotic regimes. 
Although the results obtained in this paper answer positively the basic question raised in the Introduction, they also bring new and more subtle questions to our attention, such as:

- Is it possible to identify other chaotic evolutionary attractors through mathematical models?

- Is it possible to dig into field and laboratory evolutionary time series and detect the footprint of deterministic chaos?

The answer to the first question is certainly positive, though it might be hard to discover new strange attractors. In a sense, we have already suggested how one could proceed. Good candidate models are those obtained by adding one species to any two-species interaction in which cyclic Red Queen dynamics have already been detected (e.g., the mutualistic interaction described in [Ferrière et al., 2002; Dercole, 2005]). Then one could proceed using the mix of intuition and theory described in the previous section, or, alternatively, perform the bifurcation analysis of the three-dimensional canonical equation, thus having higher chances to detect routes to chaos.

As for the second question, we suspect that scarcity of data, both in quantitative and qualitative terms, and exogenous randomness due, for example, to climatic variations, will prevent one from obtaining statistically significant answers. However, we believe it will be even more unlikely that one could prove the opposite, namely that there is no trace of endogenously produced evolutionary chaos in nature.

Finally, many are the conceptual and practical consequences of evolutionary chaos in biology (to be discussed in a forthcoming biologically-oriented paper). Chaotic Red Queen implies that even when the forces of natural selection are strong and deterministic, evolutionary trajectories may not be predicted beyond a short evolutionary time. This runs counter to the stabilizing role commonly attributed to coevolution in ecological communities, and reveals that adaptive processes may not be as predictable and replicable as previously thought. Important implications for questions of paramount interest include pathogen unpredictable evolution, the maintenance of genetic diversity in homogenized landscapes, and the process of speciation. 


\section{References}

Abarbanel, H. D. I. [1996] Analysis of Observed Chaotic Data (Springer-Verlag, New York).

Abrams, P. A. [1992] "Adaptive foraging by predators as a cause of predator-prey cycles," Evolutionary Ecology 6, 56-72.

Abrams, P. A. \& Matsuda, H. [1997] "Prey evolution as a cause of predator-prey cycles," Evolution 51, $1742-1750$.

Allgower, E. L. \& Georg, K. [1990] Numerical Continuation Methods: An Introduction (Springer-Verlag, Berlin).

Alligood, K. T., Sauer, T. D. \& Yorke, J. A. [1996] Chaos: An Introduction to Dynamical Systems (SpringerVerlag, New York).

Barnosky, A. [2001] "Distinguishing the effects of the Red Queen and Court Jester on Meiocene mammal evolution in the northern Rocky Mountains," Journal of Vertebrate Paleontology 21, 172-185.

Baumgartner, T. R., Soutar, A. \& Ferreira-Bartrina, V. [1992] "Reconstruction of the history of Pacific sardine and northern anchovy populations over the past two millennia from sediments of the Santa Barbara basin, California," California Cooperative Oceanic Fisheries Investigations Reports 33, 24-40.

Brown, J. S. \& Vincent, T. L. [1987] "A theory for the evolutionary game,” Theoretical Population Biology 31, 140-166.

Candaten, M. \& Rinaldi, S. [2000] "Peak-to-peak dynamics: A critical survey," International Journal of Bifurcation and Chaos 10, 1805-1819.

Christiansen, F. B. [1991] "On conditions for evolutionary stability for a continuously varying character," The American Naturalist 138, 37-50.

Cohen, J. E., Pimm, S. L., Yodzis, P. \& Saldan, J. [1993] "Body sizes of animal predator and animal prey in food webs," Journal of Animal Ecology 62, 67-78.

Dercole, F. [2003] "Remarks on branching-extinction evolutionary cycles," Journal of Mathematical Biology 47, 569-580. 
Dercole, F. [2005] "Border collision bifurcations in the evolution of mutualistic interactions," International Journal of Bifurcation and Chaos 15, 2179-2190.

Dercole, F., Ferrière, R. \& Rinaldi, S. [2002] "Ecological bistability and evolutionary reversals under asymmetrical competition," Evolution 56, 1081-1090.

Dercole, F., Gragnani, A., Ferrière, R. \& Rinaldi, S. [2006] "Coevolution of slow-fast populations: An application to prey-predator systems," Proceedings of the Royal Society of London B 273, 983-990.

Dercole, F., Irisson, J.-O. \& Rinaldi, S. [2003] "Bifurcation analysis of a prey-predator coevolution model," SIAM Journal on Applied Mathematics 63, 1378-1391.

Dercole, F. \& Rinaldi, S. [2008] Analysis of Evolutionary Processes: The Adaptive Dynamics Approach and its Applications (Princeton University Press, Princeton, NJ).

Dieckmann, U. \& Law, R. [1996] "The dynamical theory of coevolution: A derivation from stochastic ecological processes," Journal of Mathematical Biology 34, 579-612.

Dieckmann, U., Marrow, U. \& Law, R. [1995] "Evolutionary cycling in predator-prey interactions: Population dynamics and the Red Queen," Journal of Theoretical Biology 176, 91-102.

Doebeli, M. \& Ruxton, G. D. [1997] "Evolution of dispersal rates in metapopulation models: Branching and cyclic dynamics in phenotype space," Evolution 51, 1730-1741.

Doedel, E. J., Champneys, A. R., Fairgrieve, T. F., Kuznetsov, Yu. A., Oldeman, B., Paffenroth, R. C., Sandstede, B., Wang, X. J. \& Zhang, C. H. [2007] AUTO-07p: Continuation and bifurcation software for ordinary differential equations, Department of Computer Science, Concordia University, Montreal, QC.

Dybdahl, M. F. \& Storfer, A. [2003] "Parasite local adaptation: Red Queen versus Suicide King," Trends in Ecology \& Evolution 18, 523-530.

Feigenbaum, M. J. [1980] "The metric universal properties of period doubling bifurcations and the spectrum for a route to turbulence," Annals of the New York Academy of Sciences 357, 330-336.

Ferrière, R. [2000] "Adaptive responses to environmental threats: Evolutionary suicide, insurance and rescue,” Options Spring 2000, 12-16, International Institute for Applied Systems Analysis, Laxenburg, Austria. 
Ferrière, R., Bronstein, J. L., Rinaldi, S., Law, R. \& Gauduchon, M. [2002] "Cheating and the evolutionary stability of mutualisms," Proceedings of the Royal Society of London B 269, 773-780.

Fisher, R. A. [1930] The Genetical Theory of Natural Selection (Clarendon Press, Oxford, UK).

Gatto, M. [1990] "A general minimum principle for competing populations: Some ecological and evolutionary consequences," Theoretical Population Biology 37, 369-388.

Gavrilets, S. [1997] "Coevolutionary chase in exploiter-victim systems with polygemic characters," Journal of Theoretical Biology 186, 527-534.

Geritz, S. A. H., Kisdi, E., Meszéna, G. \& Metz, J. A. J. [1998] "Evolutionarily singular strategies and the adaptive growth and branching of the evolutionary tree," Evolutionary Ecology 12, 35-57.

Geritz, S. A. H., Metz, J. A. J., Kisdi, E. \& Meszéna, G. [1997] “The dynamics of adaptation and evolutionary branching," Physical Review Letters 78, 2024-2027.

Hastings, A. \& Powell, T. [1991] "Chaos in a three species food chain," Ecology 72, 896-903.

Hayashi, C., Ueda, Y., Akamatsu, N. \& Itakura, H. [1970] "On the behavior of self-oscillatory systems with external force," Electronics \& Communication in Japan 53-A, 150-158, (in Japanese).

Hofbauer, J. \& Sigmund, K. [1990] “Adaptive dynamics and evolutionary stability," Applied Mathematical Letters 3, 75-79.

Iwasa, Y. \& Pomiankowski, A. [1995] "Continual change in mate preferences,” Nature 377, 420-422.

Iwasa, Y. \& Pomiankowski, A. [1999] “Good parent and good genes models of handicap evolution,” Journal of Theoretical Biology 200, 97-109.

Kelley, P. H., Kowalewski, M. \& Hansen, T. A. (eds.) [2003] Predator-Prey Interaction in the Fossil Record (Plenum, New-York).

Khibnik, A. I. \& Kondrashov, A. S. [1997] “Three mechanisms of Red Queen dynamics," Proceedings of the Royal Society of London B 264, 1049-1056.

Kisdi, E., Jacobs, F. J. A. \& Geritz, S. A. H. [2001] "Red Queen evolution by cycles of evolutionary branching and extinction," Selection 2, 161-176. 
Lorenz, E. N. [1963] "Deterministic nonperiodic flow," Journal of the Atmospheric Sciences 20, 130-141.

Madan, R. (ed.) [1993] Chua's Circuit: A Paradigm for Chaos (World Scientific, Singapore).

Marrow, P., Dieckmann, U. \& Law, R. [1996] "Evolutionary dynamics of predator-prey systems: An ecological perspective," Journal of Mathematical Biology 34, 556-578.

Marrow, P., Law, R. \& Cannings, C. [1992] "The coevolution of predator-prey interactions: ESSs and Red Queen dynamics," Proceedings of the Royal Society of London B 250, 133-141.

Metz, J. A. J., Geritz, S. A. H., Meszéna, G., Jacobs, F. J. A. \& van Heerwaarden, J. S. [1996] “Adaptive dynamics: A geometrical study of the consequences of nearly faithful reproduction," in "Stochastic and Spatial Structures of Dynamical Systems,” eds. van Strien, S. J. \& Verduyn Lunel, S. M. (Elsevier Science, Burlington, MA), pp. 183-231.

Metz, J. A. J., Nisbet, R. M. \& Geritz, S. A. H. [1992] "How should we define fitness for general ecological scenarios?" Trends in Ecology \& Evolution 7, 198-202.

Ott, E., Sauer, T. D. \& Yorke, J. A. [1994] Coping with Chaos: Analysis of Chaotic Data and the Exploitation of Chaotic Systems (Wiley, New York).

Ramasubramanian, K. \& Sriram, M. S. [2000] "A comparative study of computation of Lyapunov spectra with different algorithms," Physica D 139, 72-86.

Rössler, O. E. [1976] “An equation for continuous chaos,” Physics Letters A 57, 397-398.

Schwartz, I. B. \& Smith, H. L. [1983] "Infinite subharmonic bifurcations in an SEIR epidemic model," Journal of Mathematical Biology 18, 233-253.

Soutar, A. \& Isaacs, J. D. [1974] "Abundance of pelagic fish during the 19th and 20th centuries as recorded in anaerobic sediment off California," Fishery Bulletin 72, 257-274.

Van Valen, L. [1973] “A new evolutionary law,” Evolutionary Theory 1, 1-30.

Vincent, T. L., Cohen, Y. \& Brown, J. S. [1993] "Evolution via strategy dynamics," Theoretical Population Biology 44, 149-176. 
Ziman, J. (ed.) [2000] Technological Innovation as an Evolutionary Process (Cambridge University Press, Cambridge, UK). 


\section{Figure captions}

\section{Figure 1}

The evolution set $\mathcal{X}$ characterizing short-term stationary coexistence of the food chain at the demographic equilibrium (2) (bounded volume, A), and an evolutionary trajectory tending toward a stable equilibrium of the canonical equation (4) (B). Parameter values are $r=0.5, d_{2}=0.05, d_{3}=0.02, e_{2}=0.14, e_{3}=0.14$, $c_{1}=0.5, c_{2}=3, c_{0}=0, a_{21}=0.22, a_{22}=0.25, a_{23}=0.6, a_{24}=0, a_{25}=0.04, a_{31}=0.22$, $a_{32}=0.25, a_{33}=0.6, a_{34}=0, a_{35}=-0.04, k_{1}=0.15, k_{2}=1, k_{3}=1$. Ancestral conditions in $\mathrm{B}$ are $x_{1}(0)=-0.0411, x_{2}(0)=-0.0372, x_{3}(0)=0.0075$.

\section{Figure 2}

Evolutionary strange attractor (A) and corresponding time series of resource $\left(x_{1}\right)$, consumer $\left(x_{2}\right)$, and predator $\left(x_{3}\right)$ traits (B). Parameter values as in Fig. 1 except for $k_{1}=0.64$.

\section{Figure 3}

Peak-to-peak plot of the predator trait $x_{3}$. Each point represents a pair of consecutive peaks of an $x_{3}$ time series associated with the strange attractor of Fig. 2. The curve drawn through the points can be used to forecast the next peak on the basis of the last peak.

\section{Figure 4}

Period-doubling bifurcation: a stable cycle (solid trajectory in A) becomes unstable (dashed trajectory in B) and a new stable cycle, tracing twice the bifurcating cycle, appears (solid trajectory in B). Immediately before the bifurcation (A) the attractor is a cycle with period $T$, while immediately after the bifurcation (B) the attractor is a cycle with period $2 T$.

\section{Figure 5}

A sequence of evolutionary attractors obtained for increasing values of the resource mutational rate $k_{1}$ : (A) $k_{1}=0.15$, equilibrium; (B) $k_{1}=0.2$, small cycle; (C) $k_{1}=0.5$, large cycle; (D) $k_{1}=0.6$, period- 2 cycle; (E) $k_{1}=0.635$, period-4 cycle; (F) $k_{1}=0.64$, strange attractor. Other parameter values as in Fig. 1. 


\section{Figure 6}

The standard representation of the Feigenbaum cascade obtained by plotting the peaks of $x_{3}$ within the attractor corresponding to each value of the control parameter $p$ (i.e., $k_{1}$ ). Other parameter values as in Fig. 1.

\section{Figure 7}

Two-parameter bifurcation diagram. Black lines represent bifurcation curves: Hopf and period-doubling bifurcations from left to right in the left panel. The color-code indicates the maximum (nontrivial) Lyapunov exponent associated to non-stationary evolutionary attractors; white corresponds to stable evolutionary equilibria. The region inside the blue rectangle, with coordinates $\left(z_{1}, z_{2}\right)$, is magnified and vertically stretched in the right panel. 

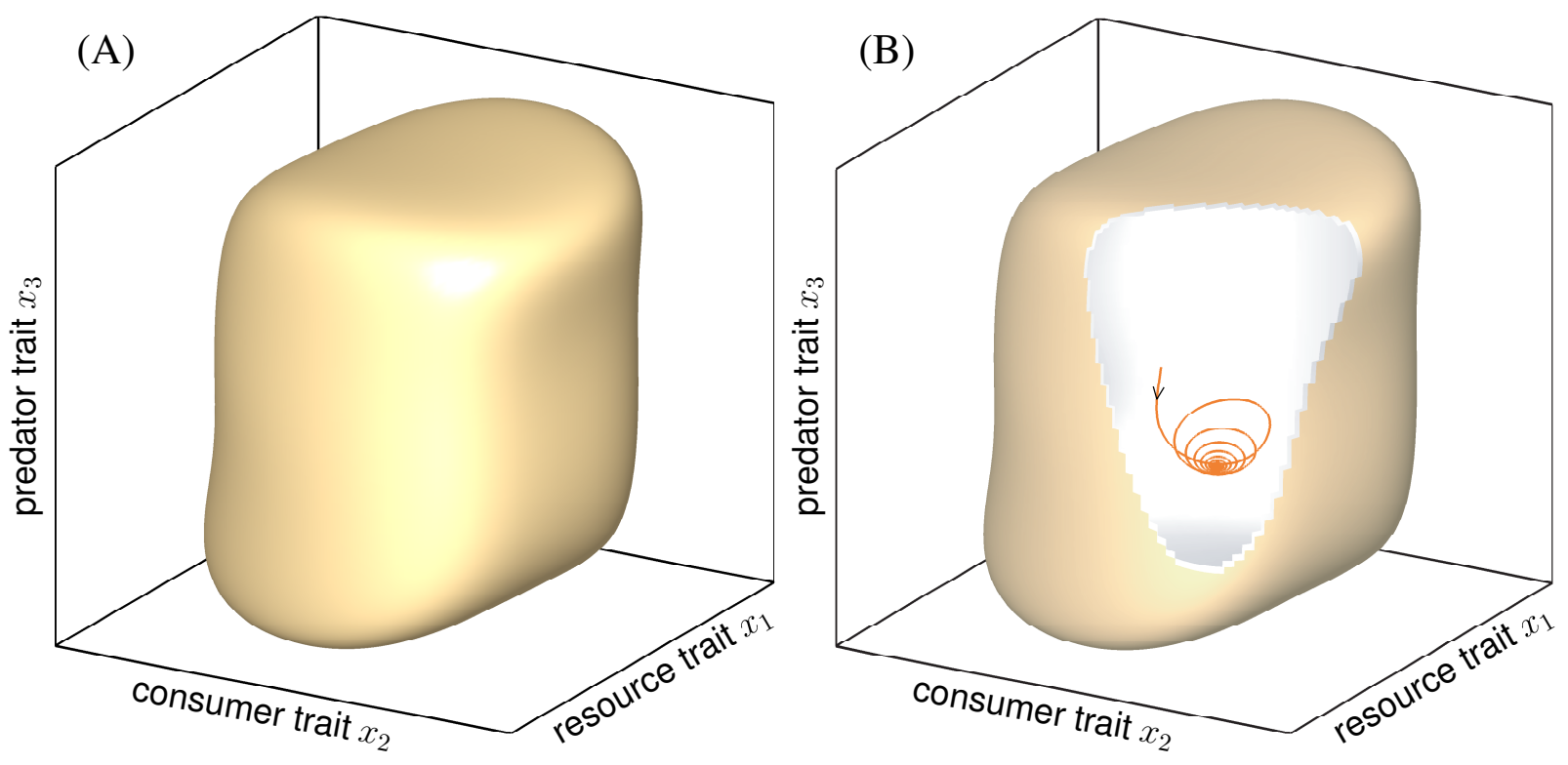

Figure 1 


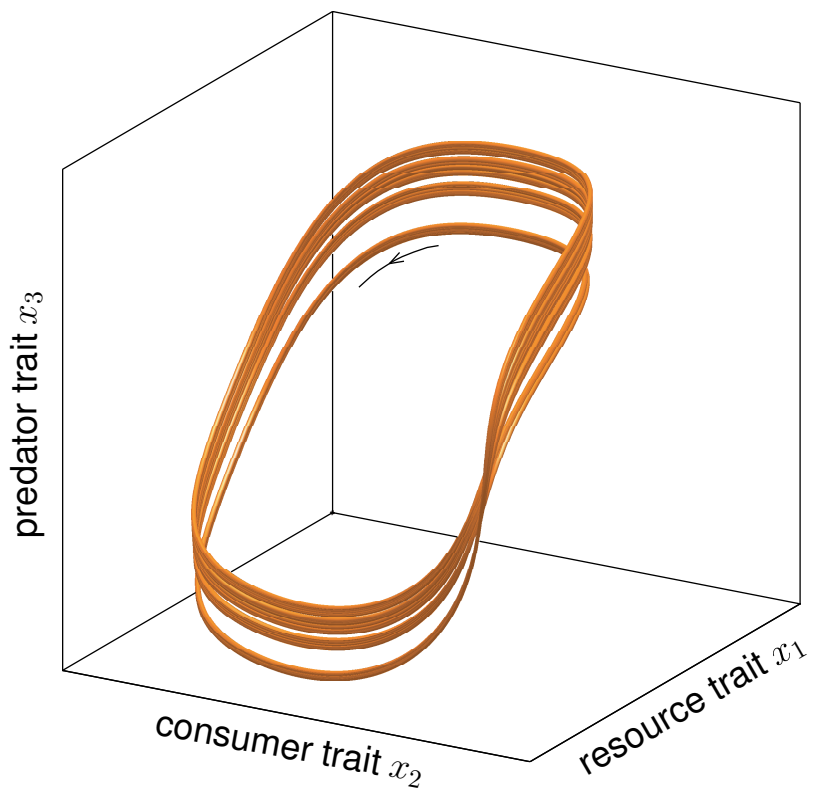

(A)

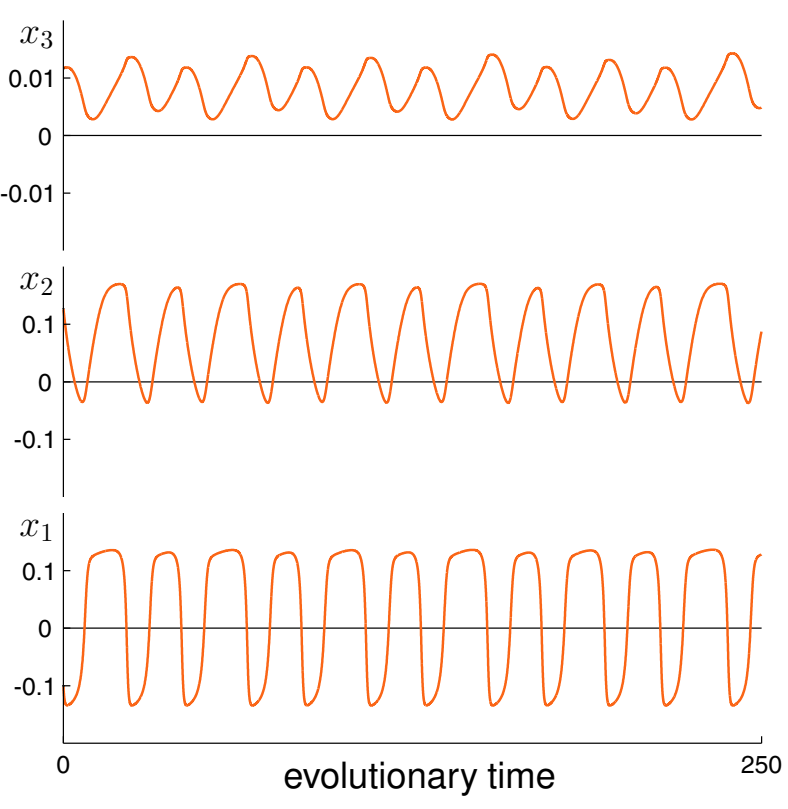

(B)

Figure 2 


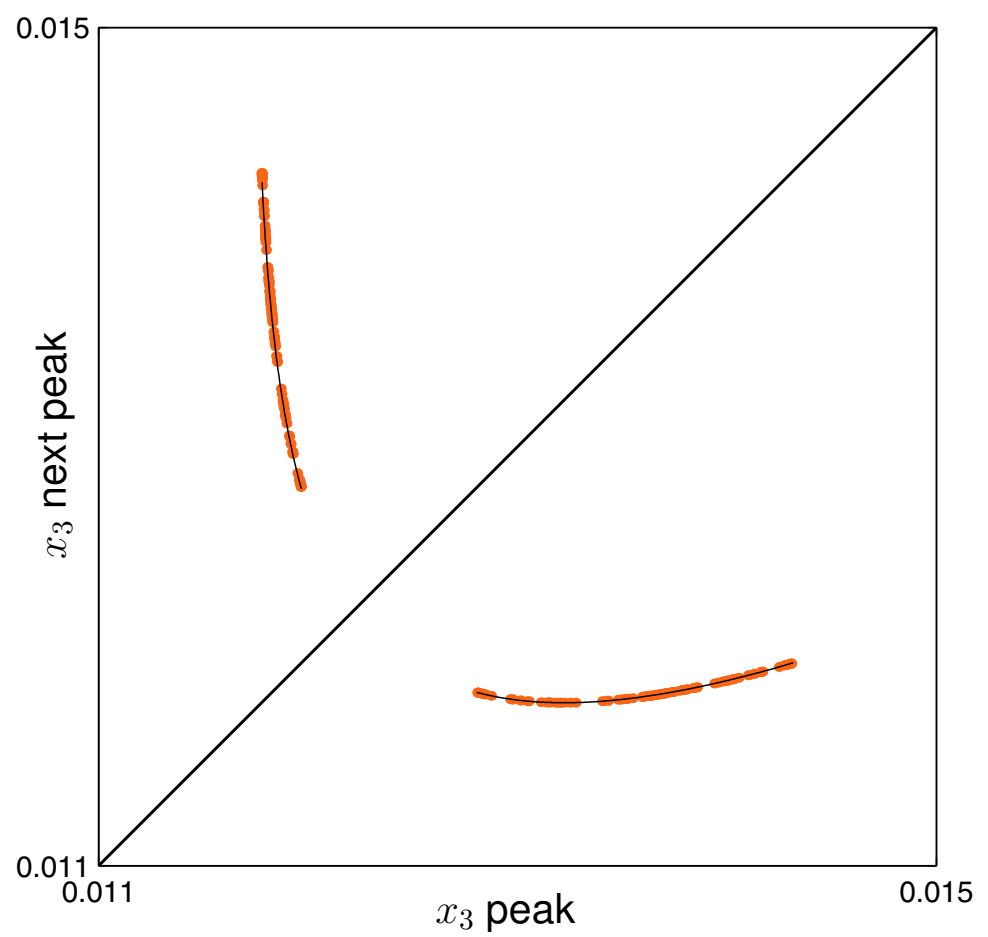

Figure 3 


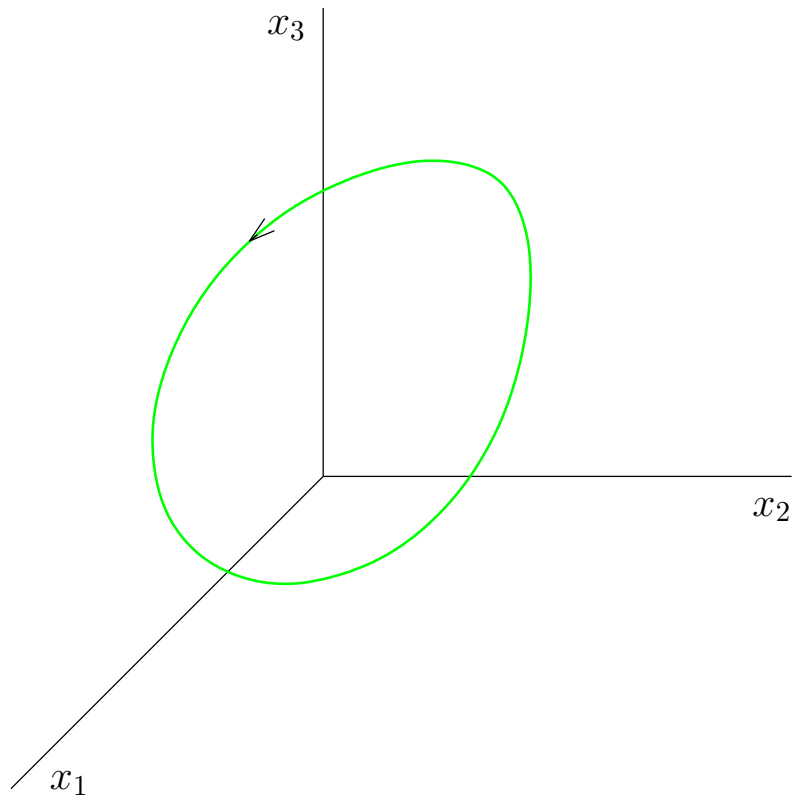

(A)

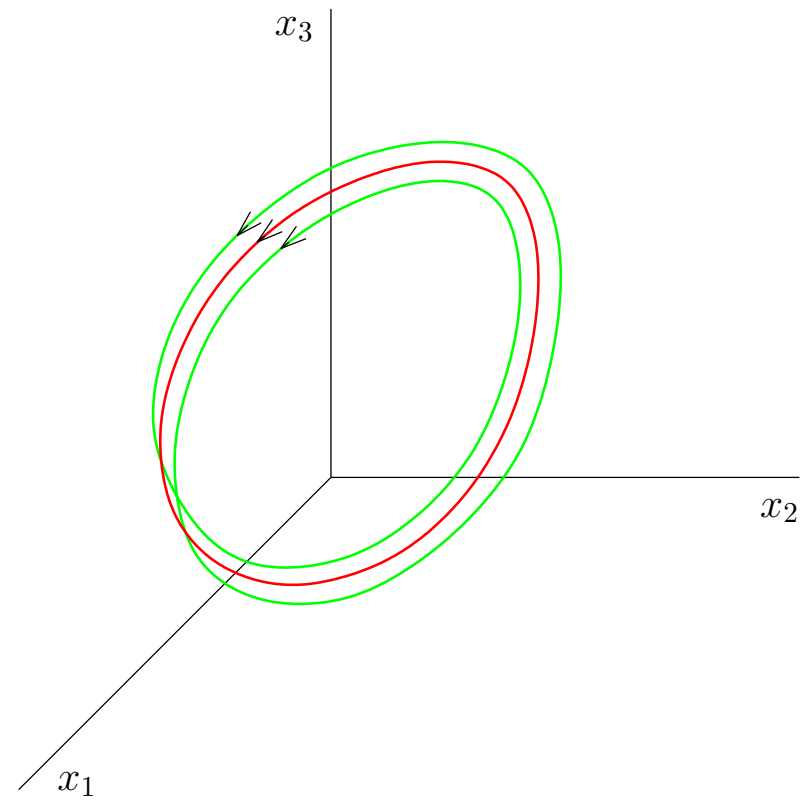

(B)

Figure 4 

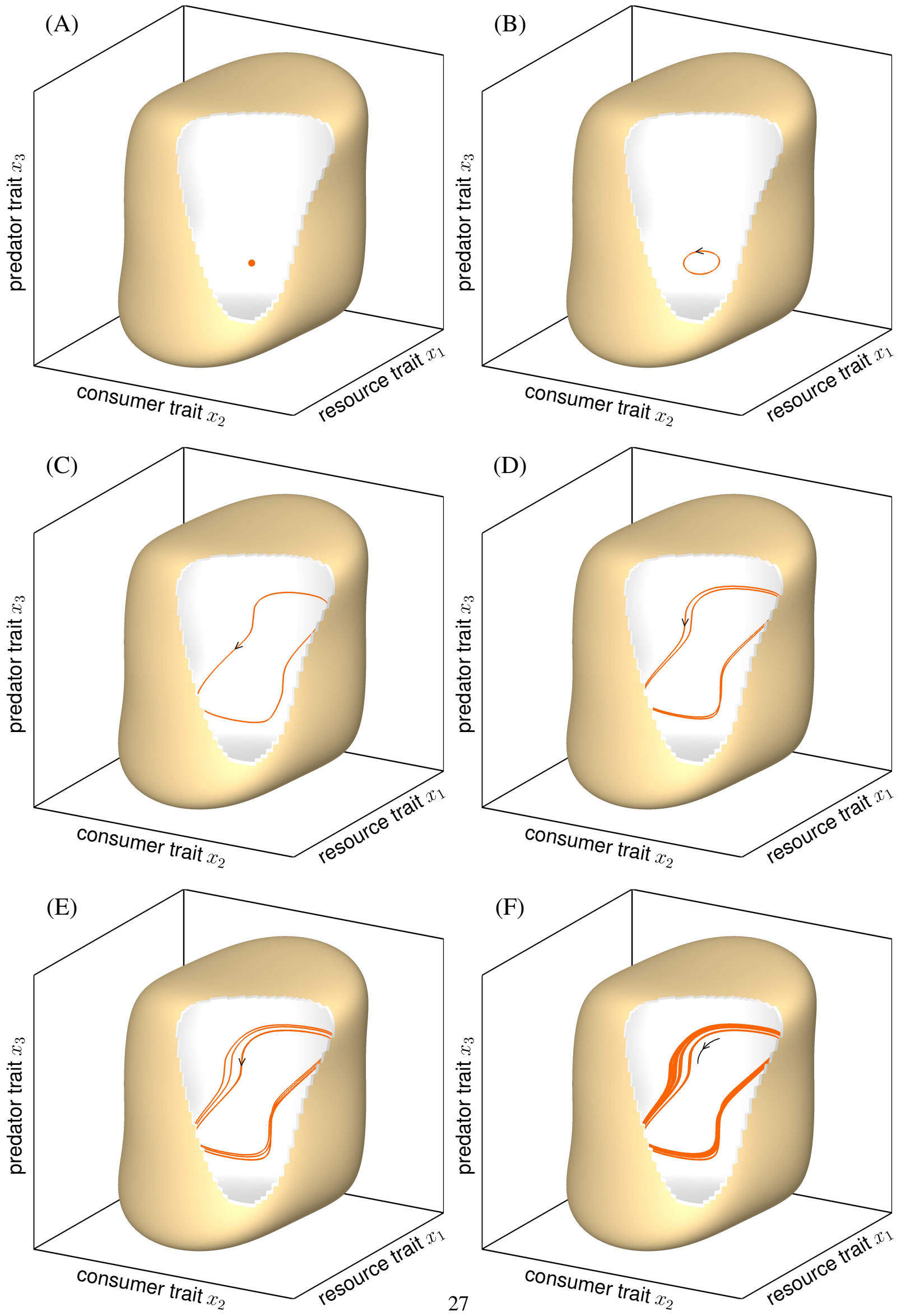

Figure 5 


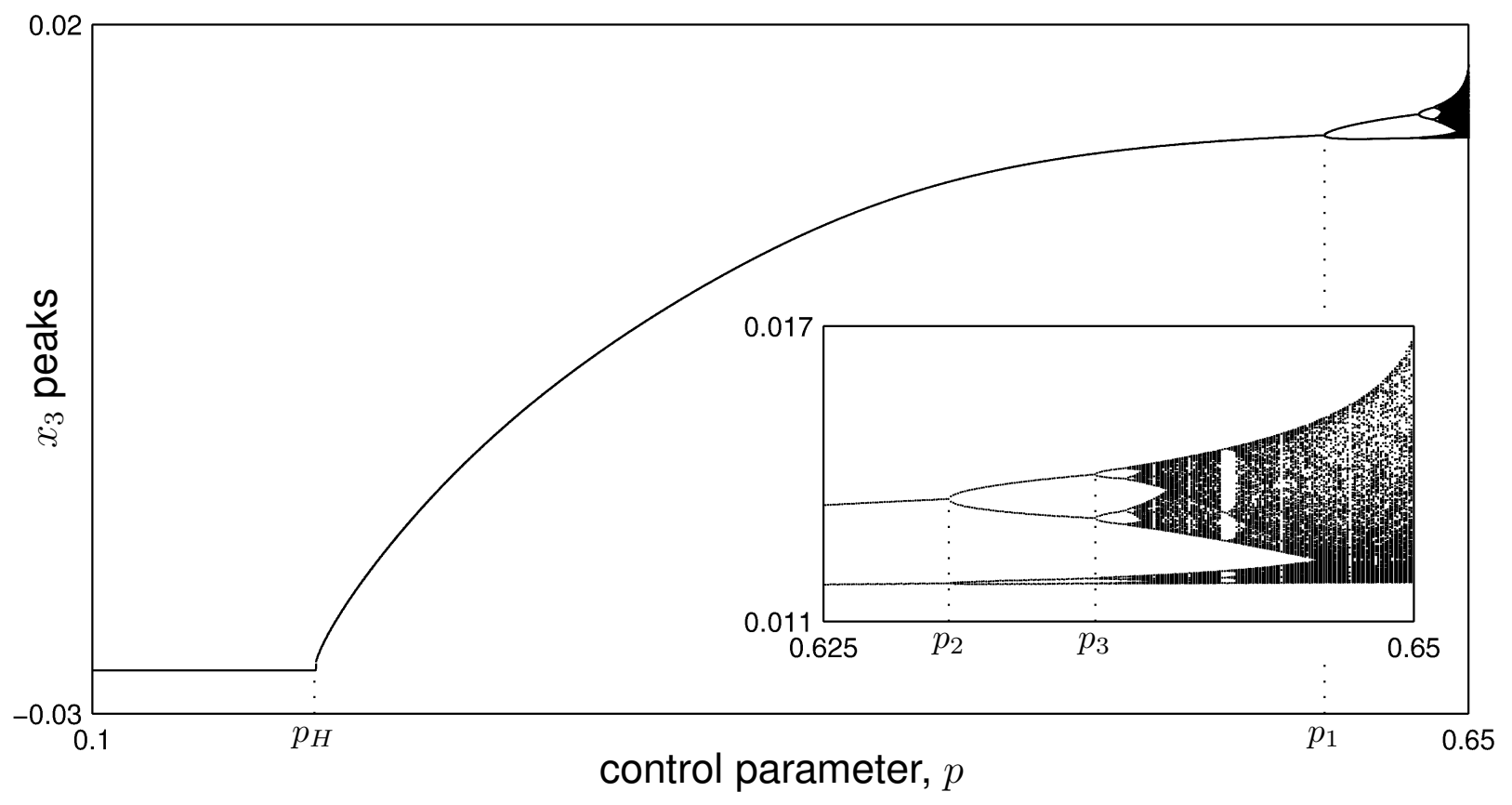

Figure 6 

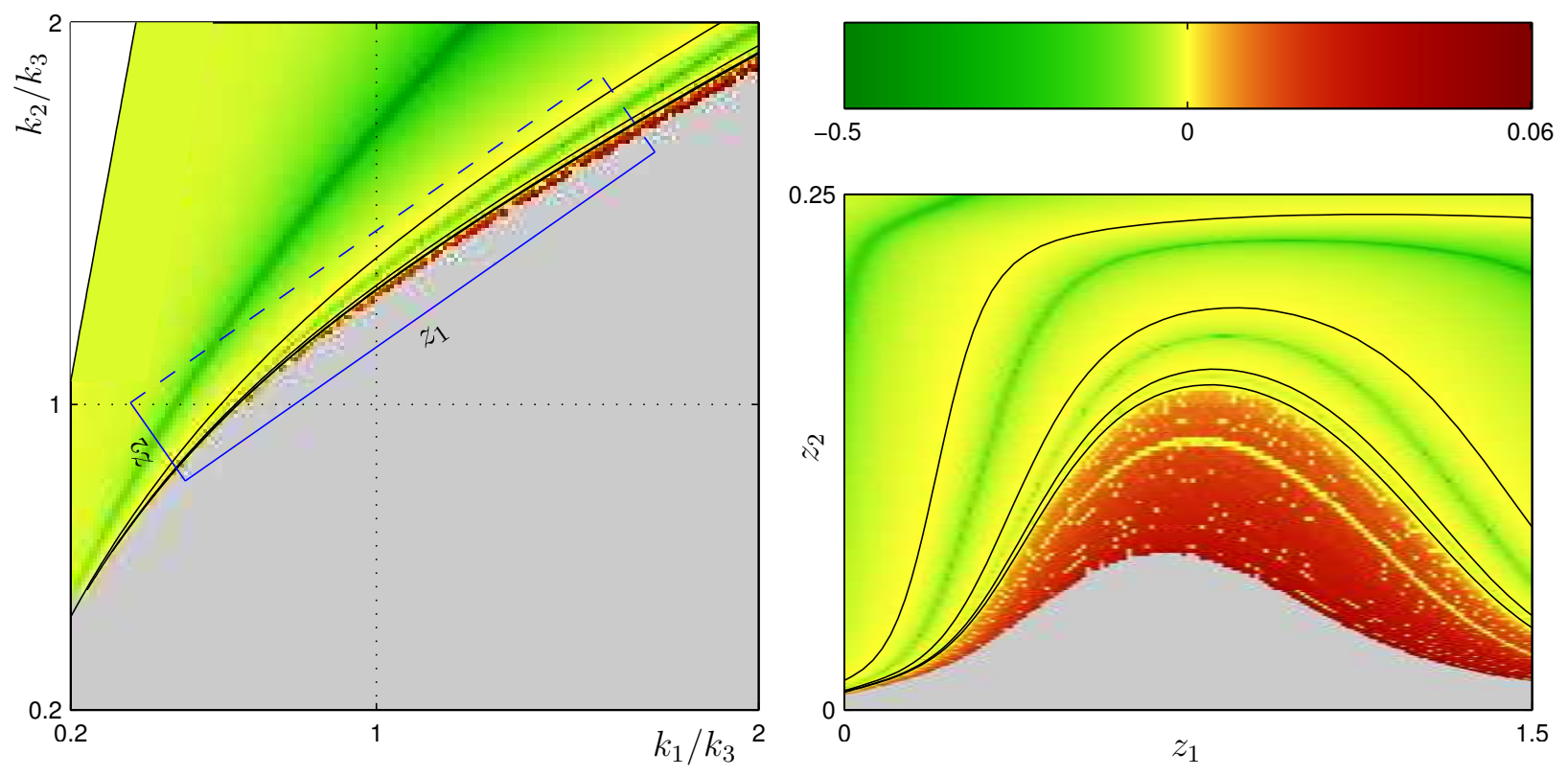

Figure 7 